\title{
Tri-block polymer with interfacial layer formation ability and its use in maintaining supersaturated drug solution after dissolution of solid dispersions
}

This article was published in the following Dove Press journal:

International Journal of Nanomedicine

\section{Ji-Jun $\mathrm{Fu}^{\prime}$ \\ Cheng-Cheng Liu \\ 'Key Laboratory of Molecular Target \& Clinical Pharmacology, School of Pharmaceutical Science, The Fifth Affiliated Hospital of Guangzhou Medical University, Guangzhou Medical University, Guangzhou, People's Republic of China; ${ }^{2}$ Department of Medical Oncology, The Fifth Affiliated Hospital of Guangzhou Medical University, Guangzhou Medical University, Guangzhou, People's Republic of China}

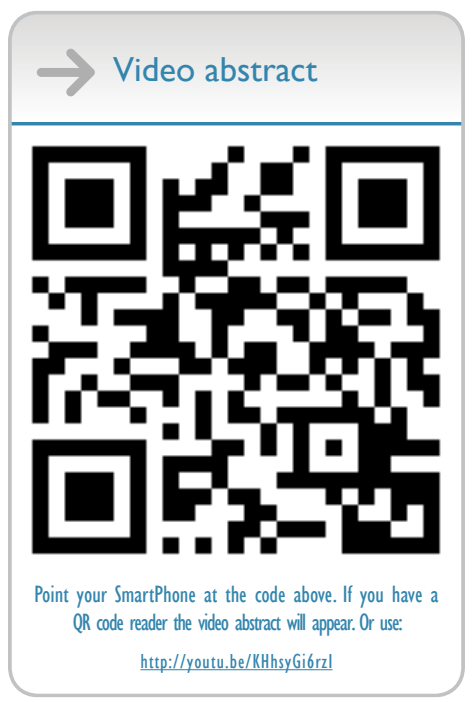

Correspondence: Cheng-Cheng Liu Department of Medical Oncology, The Fifth Affiliated Hospital of Guangzhou Medical University, Guangzhou Medical University, Guangzhou, Guangdong, 510700, People's Republic of China Tel +86203221 8139

Email liuchengchengfI@I63.com
Background: Maintaining a supersaturated drug solution after the dissolution of the solid dispersions of water insoluble drugs continues to be a great challenge and is important to the oral bioavailability enhancement of hardly soluble drugs.

Methods: Nimodipine solid dispersions were prepared by hot-melt extrusion and a special tri-block polymer was employed as a co-carrier. The solid dispersions were characterized by modulated differential scanning calorimetry, transmission electron microscopy, hydrogennuclear magnetic resonance and so on.

Results: The tri-block polymer was able to inhibit the formation of drug crystals after dissolution of the solid dispersions. Due to the unique interfacial layer formation ability of the tri-block polymer, a special drug loading micelle which encapsulated the compound and the hydrophobic fragments of the copolymers appeared in the release media. The tri-block polymer was composed of a hydrophilic part forming the shell of micelles, a hydrophobic part shaping the core of micelles, and a special intermediate hydrophilicity part constructing the interfacial layer of micelles.

Conclusion: The tri-block polymer was not only able to stabilize the supersaturated drug solution of solid dispersions to enhance the oral bioavailability of hardly soluble drugs, but is also a potential candidate to construct micelles for systemic administration, due to the good compatibility and organic solvents free micelle formation procedure.

Keywords: micelles, disintegrate, stability in blood circulation, solid dispersions, recrystallization

\section{Introduction}

Solid dispersions (SD) are often employed to enhance oral bioavailability of insoluble drugs. In SD, compounds exist as amorphous or molecular state. After dissolving SD in body fluids or other media, it is hoped that molecular or amorphous drugs are quickly absorbed to play therapeutic effects. However, there are several drawbacks associated with SD retarding its practical application, such as long-term storage instability, easy to be destroyed by moisture or organic solvents, as well as hard to be formulated. Until now, some reports have proposed using excipients with high glass transition temperature (Tg) to ensure long-term storage stability of SD, ${ }^{1}$ and hot-melt extrusion (HME) technique makes choice of this kind of excipient a reality. Furthermore, the SD was successfully formulated into tablets by employing direct compression technique. ${ }^{1,2}$ It seems that problems which were negatively affecting the practical application of SD are well solved now. Actually, there is still a big obstacle. After water-insoluble compound is released from SD in dissolution media or body fluids, sometimes recrystallization of the drug happens quickly. As drug crystals are difficult to be absorbed, high oral 
bioavailability would not be achieved. In the past research, storage of the SD at high temperature for several days was found to be effective in inhibiting the recrystallization process. ${ }^{3}$ However, this method is often doubted and not easy to be accepted as high storage temperature is normally assumed to be a destroying factor for SD. In a word, method of maintaining supersaturated drug solution after SD dissolution still needs to be investigated.

Nowadays, polymers and copolymers are frequently used as drug carriers, especially in nanodrug delivery systems for targeted treatment. Normally, hardly soluble compounds are entrapped in the polymers and, the two components form nanosized spheres which are well dispersed in water or other media. For the purpose of escaping reticuloendothelial system in blood circulation, the polymers used are often decorated by polyethylene glycol (PEG) or other water-soluble materials. The insoluble drugs could be well entrapped in the polymers and do not recrystallize from the nanoemulsions. This phenomenon clues that compounds released from the SD could exist as stable nanosized particles in media if suitable polymers are selected as SD excipients. The polymers must be able to encapsulate the released drug molecules spontaneously and then form nanosized particles after immersing in water immediately.

The aim of the present study was to propose a unique tri-block polymer named polyoxyethylene/vinyl acetate/ $\mathrm{N}$-vinyl-caprolactam (PEO-VA-NVCL) with interfacial formation ability and use it as SD excipient. By doing this, supersaturated drug solution maintained as the polymer was able to entrap the released drug molecules and form nanosized micelles spontaneously after immersing in water immediately. In other words, nanotechnology was employed to overcome an important problem of SD and, a kind of micelle material with excellent performances was suggested.

Nimodipine (NM) with extremely low water solubility was chosen as a model drug.

\section{Methods}

\section{Characterization of PEO-VA-NVCL}

PEO-VA-NVCL was gifted by a friend. Its molecular weight was measured by gel permeation chromatography with tetrahydrofuran as solvent. Infrared spectroscopy of the polymer was also obtained.

Add suitable amount of the polymer to water while stirring to get micelle solution, the final concentration of the polymer was $10 \mathrm{mg} / \mathrm{mL}$. The micelle solution was photographed and, the particle size was measured by dynamic light scattering (DLS) method (Malvern).
Dissolving PEO-VA-NVCL in $\mathrm{D}_{2} \mathrm{O}$ and $\mathrm{CDCl}_{3}$ to getmicelle solution and clear solution separately, then hydrogen-nuclear magnetic resonance ( $\left.{ }^{1} \mathrm{H}-\mathrm{NMR}\right)$ spectroscopy of the polymer was measured to get the information about the structure.

\section{Preparation of NM-SD by HME}

Two kinds of NM-SD were prepared by HME. The weight ratio of NM, polyvinyl pyrrolidone vinyl acetate (PVP-VA) (Kollidon VA64, BASF Corp.) and PEO-VA-NVCL were 15: 85:0 and 15:75:10 separately. The extrusion temperature was $180^{\circ} \mathrm{C}$. All extrudes were milled to powder.

\section{Characterization of the NM-SD}

Modulated differential scanning calorimetry was used to measure the $\mathrm{Tg}$ of the two kinds of NM-SD. The heating rate was $2^{\circ} \mathrm{C} / \mathrm{min}$.

Dissolution test was carried out according to United States Pharmacopeia. The dissolution media was water and, the volume was $900 \mathrm{~mL}$. The amount of NM-SD added was $200 \mathrm{mg}$.

Add the NM-SD $(10 \mathrm{mg})$ to water $(1 \mathrm{~mL})$ to get supersaturated solutions of NM, then keep the solutions at room temperature for $8 \mathrm{~h}$. The solution obtained from NM-SD containing PVP-VA only was photographed by microscopy. The solution obtained from NM-SD containing PEO-VA-NVCL was scanned by transmission electron microscopy (TEM) and, the particle size of the micelle was measured by DLS method.

NM-SD containing PEO-VA-NVCL was dissolved in $\mathrm{D}_{2} \mathrm{O}$ and $\mathrm{CDCl}_{3}$ to get micelle solution and clear solution separately, then ${ }^{1} \mathrm{H}-\mathrm{NMR}$ spectroscopy of the solutions was measured to get structural information of the micelles.

\section{Hemolysis test}

The study protocol was approved by the Ethics Committee of Guangzhou Medical University.

The $1 \mathrm{~mL}$ rat blood was diluted by phosphate-buffered saline (PBS) and centrifugated at $800 \mathrm{rpm}$ for $5 \mathrm{~min}$, the process was repeated for two more times to remove blood serum. Then, the blood cells were suspended in PBS and, the total volume was $1 \mathrm{~mL}$. Around $100 \mu \mathrm{L}$ blood cells in PBS was added to $900 \mu \mathrm{L}$ of the triblock polymer solution in PBS, thus the final concentration of the polymer was 10 , 20, 50, 100, 200, 500, and 1,000 $\mu \mathrm{g} / \mathrm{mL}$. PBS and pure water were used as negative and positive control respectively. The samples were left undisturbed at room temperature for 1 day, the absorbance of the supernatants was measured at $540 \mathrm{~nm}$ to calculate the hemolysis rate. The particle size of the supernatants was also measured to evaluate the stability of the polymer micelles in the blood. 


\section{Results}

\section{Features of the tri-block polymer}

As shown in Figure 1A, the average molecular weight of the tri-block polymer was $11,200 \mathrm{~g} / \mathrm{mol}$. The infrared spectroscopy (Figure 1B) corresponded well to the chemical structure of the polymer (Figure 1D).

The tri-block polymer was composed of three fragments, the PEO fragment, the VA fragment, and the NVCL fragment. Actually, the molar ratio of the three fragments was 13:30:57. As is widely known, PEO fragment is water soluble and VA fragment is hydrophobic. Due to the amphipathic property of the polymer, it was assumed that PEO-VA-NVCL was a potential micelle material. Actually, typical micelle solution would appear simply by adding the polymer granules to water and stirring for a few minutes (Figure 1C). The average particle size of the micelles was measured to be about $64 \mathrm{~nm}$ (Figure 2). Additionally, there was only one peak in the particle sizing graph, which indicated that the micelle particles were quite uniform. Despite great advances in nanodrug delivery systems and hundreds of amphipathic polymers proposed, ideal materials were still on the way. In order to prepare micelle solution, organic solvents and complicated handling procedures are required by almost all the polymers reported. But it was not the case for PEO-VA-NVCL, the micelle formation process of the tri-block polymer was free from organic solvents and amazingly simple. The uniform micelle particle obtained and the environmental friendly handling procedure make PEOVA-NVCL an excellent candidate to construct nanodrug delivery systems.

In order to further characterize the structure of the micelles made by the tri-block polymer, ${ }^{1} \mathrm{H}-\mathrm{NMR}$ of the micelle solution $\left(\mathrm{D}_{2} \mathrm{O}\right)$ and the clear solution $\left(\mathrm{CDCl}_{3}\right)$ were compared. As shown in Figure 2A (lower), when PEO-VA-NVCL was
A

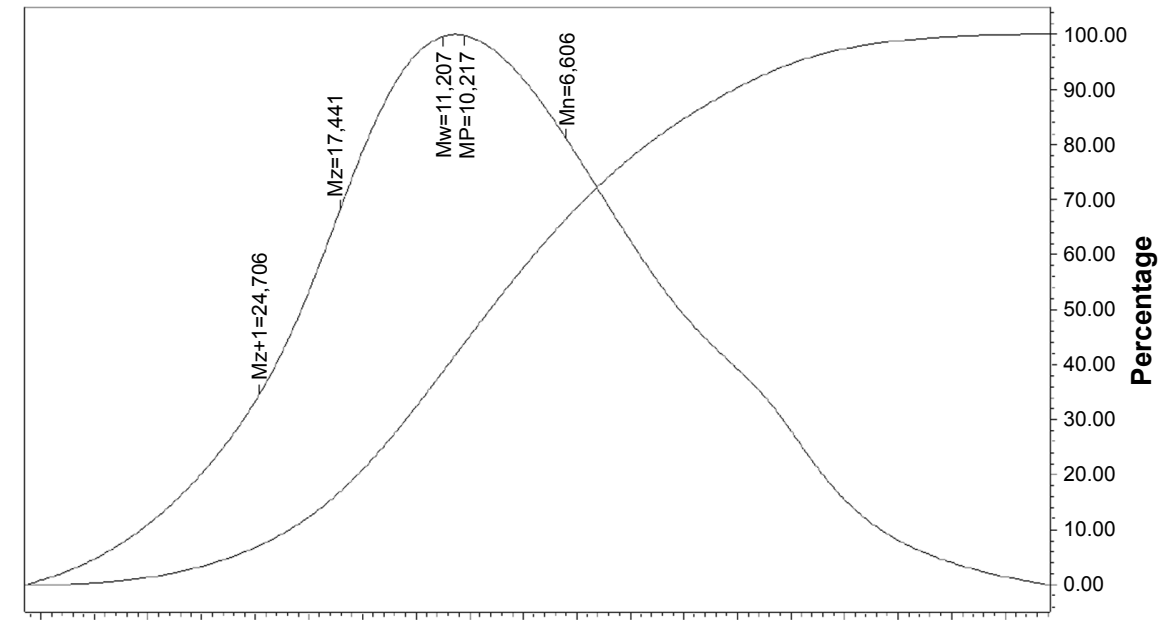

$\begin{array}{llllllllllllllllllllll}4.80 & 4.70 & 4.60 & 4.50 & 4.40 & 4.30 & 4.20 & 4.10 & 4.00 & 3.90 & 3.80 & 3.70 & 3.60 & 3.50 & 3.40 & 3.30 & 3.20 & 3.10 & 3.00\end{array}$

$\log \mathrm{MW}$

\section{B}

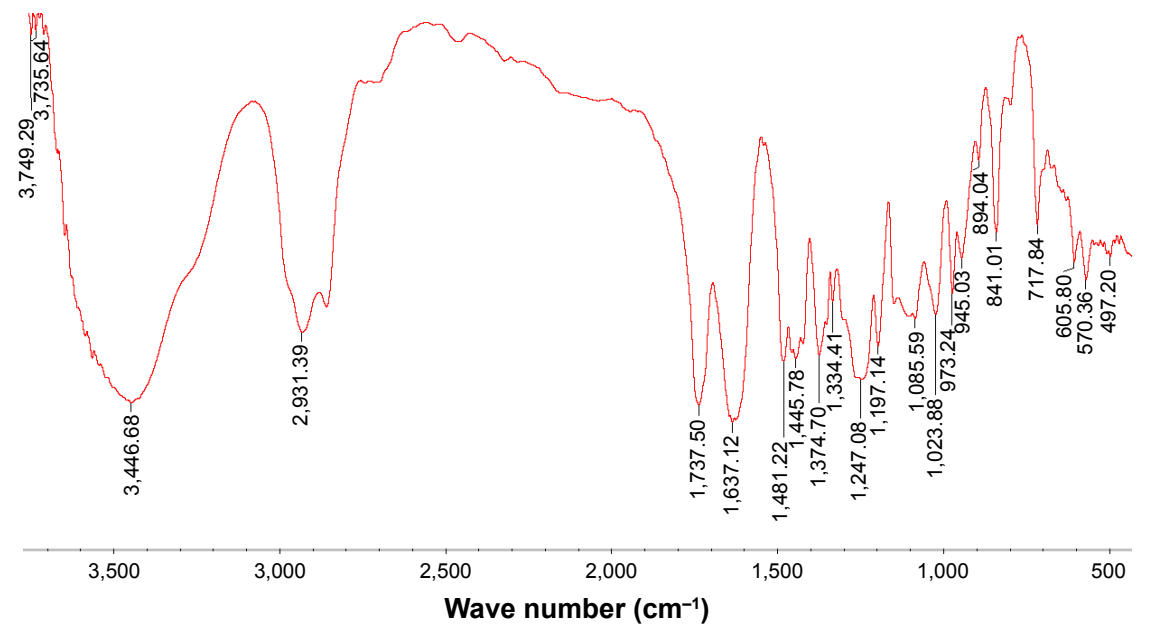

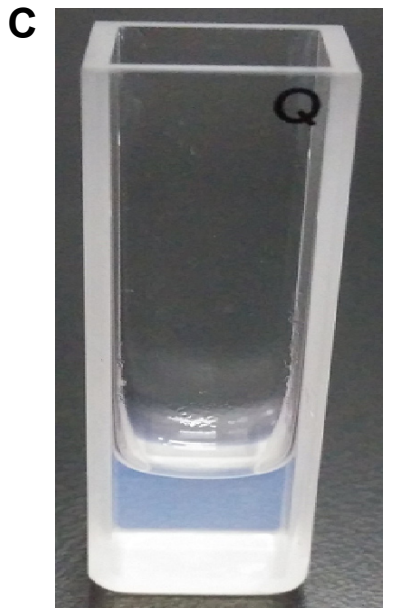

D

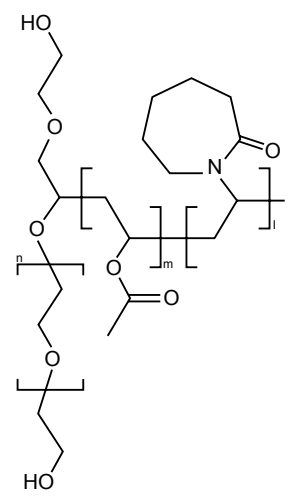

Figure I Characterization of the tri-block polymer PEO-VA-NVCL. (A) Average molecular weight of the polymer measured by GPC. (B) Infrared spectroscopy of the polymer. (C) Picture of the polymer micelle solution. (D) Chemical structure of the polymer.

Abbreviations: GPC, gel permeation chromatography; MW, molecular weight; PEO-VA-NVCL, polyoxyethylene/vinyl acetate/N-vinyl-caprolactam. 
A

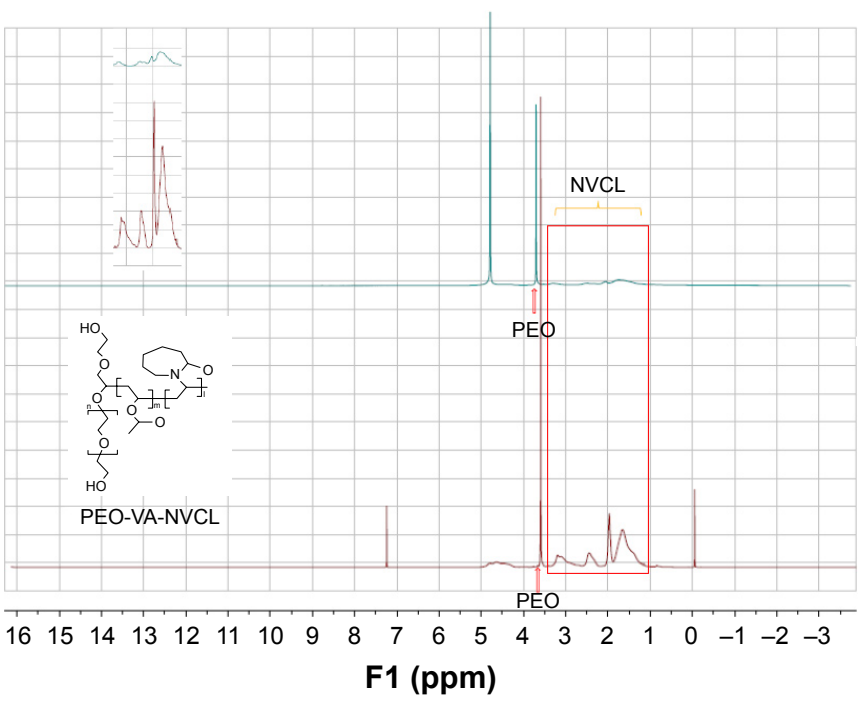

B

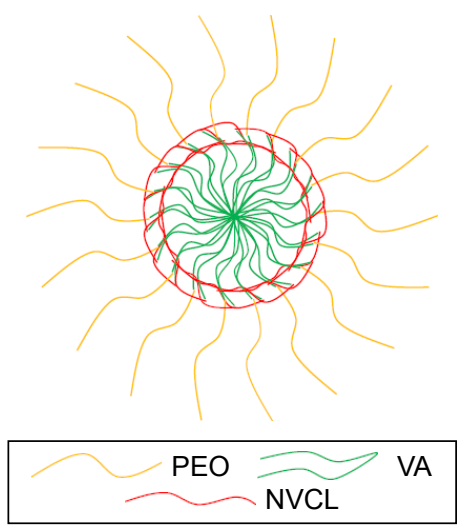

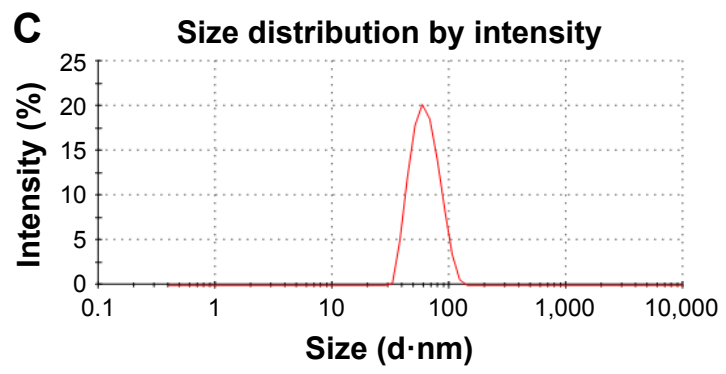

Figure 2 Structural features of the micelles constructed by the tri-block polymer PEO-VA-NVCL. (A) ' $\mathrm{H}$-NMR of the polymer dispersed in $\mathrm{D}_{2} \mathrm{O}$ (upper) and dissolved in $\mathrm{CDCl}_{3}$ (lower). (B) Schematic diagram of the polymer micelles. (C) Particle size of the polymer micelles measured by DLS.

Abbreviations: d, diameter; DLS, dynamic light scattering; 'H-NMR, hydrogen-nuclear magnetic resonance; NVCL, N-vinyl-caprolactam; PEO, polyoxyethylene; PEO-VA$\mathrm{NVCL}$, polyoxyethylene/vinyl acetate/N-vinyl-caprolactam; VA, vinyl acetate.

totally dissolved in organic solvent and all the hydrogen atoms would generate signals, both PEO signal (3.6 ppm) and NVCL signal (1.5-3.5 ppm) were observed. On the other hand, Figure 2A (upper) shows that when PEO-VANVCL was dispersed in water and only hydrogen atoms surrounded by $\mathrm{D}_{2} \mathrm{O}$ molecules would generate signals, $\mathrm{PEO}$ signal was still obvious while NVCL signal was much weaker but detectable. Without doubt, the results clued that PEO fragment was hydrophilic and, it stretched into water to form shell of the micelles. But what does the much weaker but detectable NVCL signal mean? One interpretation said that part of NVCL fragment stretched into water and part of NVCL fragment immersed into the hydrophobic core of the micelles. However, this explanation was doubtable. It was unreasonable for the same NVCL fragment to exhibit opposite hydrophilicity. A much more acceptable explanation was that the NVCL fragment located between the hydrophilic shells composed of PEO fragment and the hydrophobic core composed of VA fragment, as shown in Figure 2B. In other words, the NVCL fragment constructed a special interfacial layer of the micelle which separated the PEO shell and the
VA core. In this case, as the NVCL fragment was exposed to water to a restricted extent, the corresponding signal was still detectable but much weaker. Chemical structure of NVCL fragment is similar to that of PVP. Due to the increased hydrophobicity caused by replacement of five membered ring by seven membered ring, hydrophilicity of NVCL fragment is intermediate while that of PVP is good. Until now, no matter how many fragments are there in the micelle polymers as reported, the fragments are either hydrophilic or hydrophobic. As a result, all the suggested micelles comprise two parts, the hydrophilic shell and the hydrophobic core. The present study proposed a unique micelle with additional interfacial layer. This special structure may assign new applications to micelles containing PEO-VA-NVCL.

\section{Application of PEO-VA-NVCL in maintaining the supersaturated state of NM-SD dissolution media}

As shown in Figure 3A, the Tg of the NM-SD containing PVP-VA only was $89^{\circ} \mathrm{C}$ and, that of the NM-SD containing PEO-VA-NVCL was $82^{\circ} \mathrm{C}$. Further information which 
A

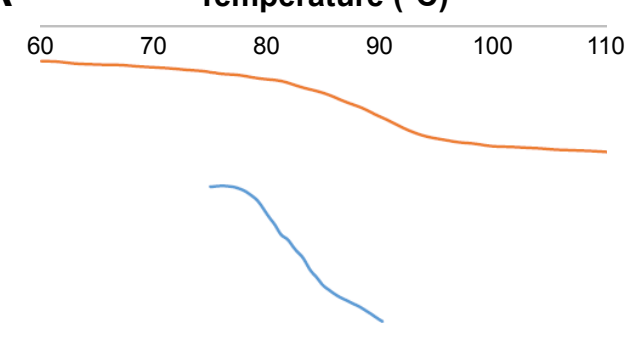

B

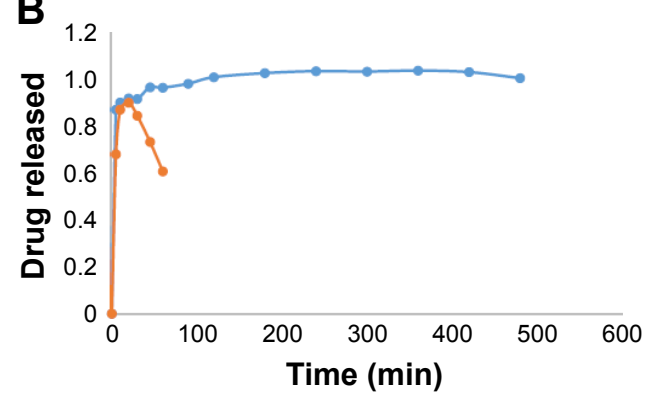

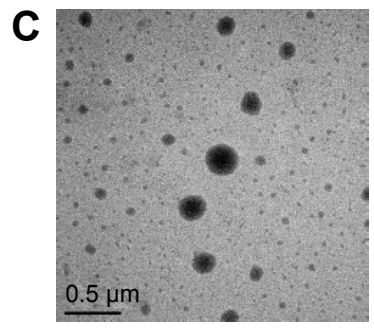

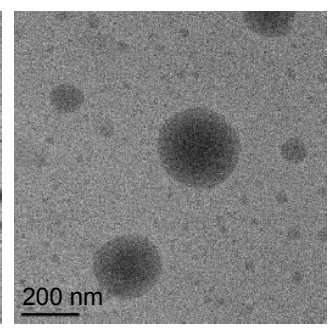

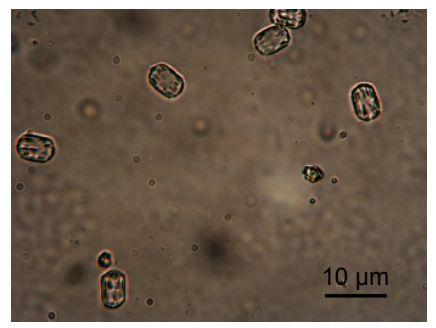

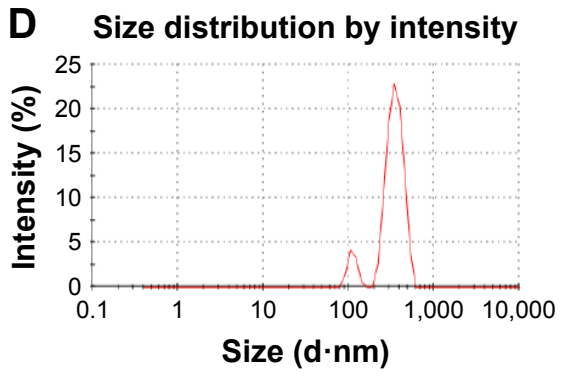

Figure 3 Characterization of the two kinds of NM-SD prepared by HME. (A) Tg of the NM-SD measured by modulated-DSC. NM-SD containing PVP-VA only (red) and NM-SD containing both PVP-VA and PEO-VA-NVCL (blue). (B) Release curves of the NM-SD containing PVP-VA only (red) and NM-SD containing both PVP-VA and PEOVA-NVCL (blue). (C) TEM images of the micelle solution prepared by dissolving the NM-SD containing both PVP-VA and PEO-VA-NVCL and microscopy image of the drug crystals in the solution obtained from dissolving NM-SD containing PVP-VA only. (D) Particle size measurement of the micelle solution prepared by dissolving the NM-SD containing both PVP-VA and PEO-VA-NVCL.

Abbreviations: d, diameter; DSC, differential scanning calorimetry; HME, hot-melt extrusion; NM-SD, nimodipine-solid dispersions; PEO-VA-NVCL, polyoxyethylene/vinyl acetate/N-vinyl-caprolactam; PVP-VA, polyvinylpyrrolidone vinyl acetate; TEM, transmission electron microscopy; Tg, glass transition temperature.

could validate the amorphous state of the NM in the SD were shown in literature. ${ }^{1-3}$

Figure 3B shows that high dissolution extent could be achieved by both NM-SD formulations. However, the release curve of the NM-SD containing PVP-VA only saw rapid decline after reaching the peak while that of the NM-SD containing PEO-VA-NVCL was steady until the end of the test. Actually, drug crystals could be observed in the former dissolution media, indicating recrystallization of the compound. On the other hand, the latter dissolution media maintained clear throughout the test. In other words, using the tri-block polymer as HME excipient at a low dose successfully inhibited drug recrystallization in release media and maintained the supersaturated condition of the compound. This feature is certainly helpful for achieving satisfactory oral bioavailability of water insoluble drugs.

In order to clarify the mechanisms behind the recrystallization inhibition effect of the tri-block polymer, suitable amount of two kinds of NM-SD was added to $1 \mathrm{~mL}$ water separately to get higher final concentration than dissolution test. Figure $3 \mathrm{C}$ shows that there were many drug crystals in the fluids obtained from NM-SD containing PVP-VA only and, the crystals were about several micrometers long. On the other hand, drug crystals were absent in the fluids obtained from NM-SD containing PEO-VA-NVCL and, the fluids appeared to be micelle solution. Actually, micelles with different sizes (ranging from $10 \mathrm{~nm}$ to $>200 \mathrm{~nm}$ ) could be observed by TEM and, the micelles showed clear core-shell structure (Figure 3C). DLS measurement revealed comparable results to TEM, there were two groups of micelles, with average particle sizes to be $115 \mathrm{~nm}$ and $480 \mathrm{~nm}$ separately (Figure 3D). All the above results indicated that the NM was entrapped into the micelles with the help of the tri-block polymer PEO-VA-NVCL during the release process of NM-SD, thus inhibiting drug recrystallization and maintaining the supersaturated solution of the compound.

Although PVP-VA seems to be an amphipathic polymer, it is totally soluble in water. As a result, there was a question: what was the drug loading micelles in the dissolution media composed of, NM with PEO-VA-NVCL only or NM with PEO-VA-NVCL and PVP-VA? To figure out the question, ${ }^{1} \mathrm{H}-\mathrm{NMR}$ of the clear solution prepared by dissolving the NM-SD containing PEO-VA-NVCL into $\mathrm{CDCl}_{3}$ and the micelle solution prepared by dispersing the same NM-SD into $\mathrm{D}_{2} \mathrm{O}$ were captured. As shown in Figure 4A (lower), NM signals were observed as predicted (6.3-8.5 ppm) when all the components were dissolved in organic solvent. But when NM-SD was dispersed in water in the form of micelles, the 


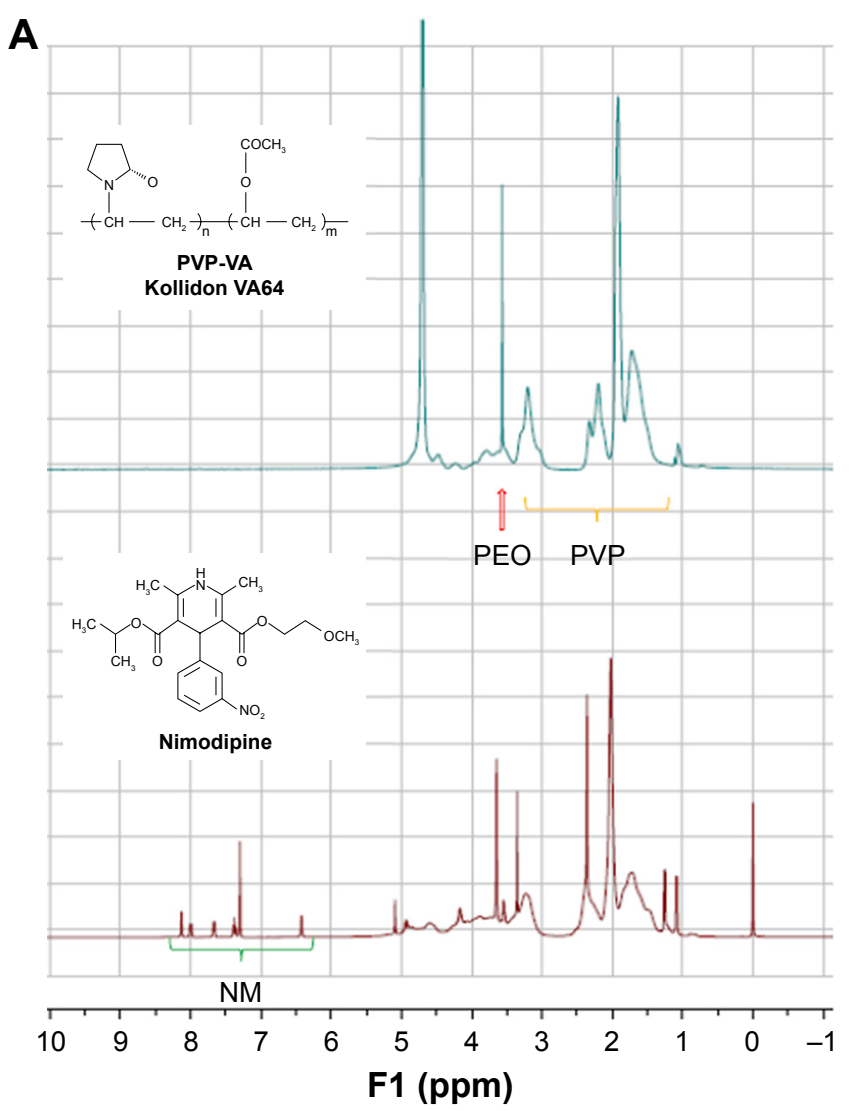

B
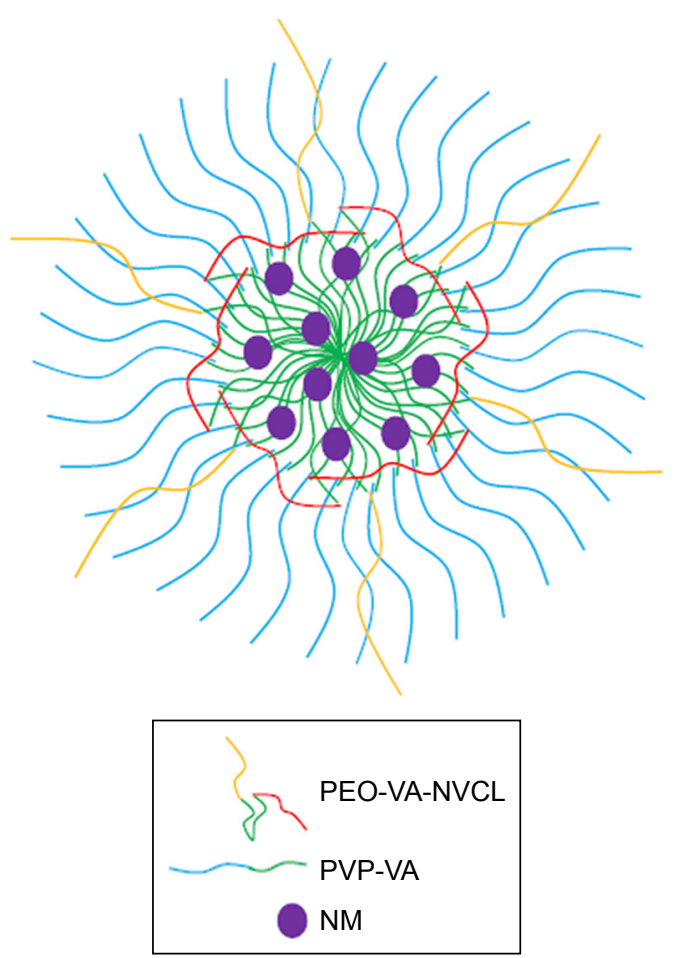

Figure 4 Structural feature of the drug loading micelles prepared by dissolving the NM-SD containing both PVP-VA and PEO-VA-NVCL. (A) 'H-NMR of the NM-SD dispersed in $\mathrm{D}_{2} \mathrm{O}$ (upper) and dissolved in $\mathrm{CDCl}_{3}$ (lower). (B) Schematic diagram of the drug loading micelles.

Abbreviations: 'H-NMR, hydrogen-nuclear magnetic resonance; NM, nimodipine; PEO-VA-NVCL, polyoxyethylene/vinyl acetate/N-vinyl-caprolactam; PVP-VA, polyvinylpyrrolidone vinyl acetate; SD, solid dispersions.

corresponding signals of NM disappeared (Figure 4A upper). The results implied that the NM was located in the core of the micelles. More importantly, both Figure 4A (lower) and Figure 4A (upper) show obvious PEO (3.6 ppm) and PVP (1.0-3.5 ppm) signals, indicating PEO together with PVP constructing the shell of the micelles. As discussed above, PVP-VA is able to be completely dissolved in water, as a result, PVP-VA solution is clear solution instead of micelle solution. That is to say, PVP-VA alone is not able to form micelles. And because of this, the NM recrystallized quickly after PVP-VA dissolved in water for the NM-SD containing PVP-VA only. However, to our surprise, PVP-VA successfully played the role of micelle-shaping material with the help of the tri-block polymer PEO-VA-NVCL and encapsulated the drug as well. Briefly, when PVP-VA and a small amount of PEO-VA-NVCL were used as co-excipients to formulate NM-SD, PEO-VA-NVCL together with PVP-VA constructed the drug loading micelles, with PEO and PVP working as the shell and VA fragment working as the drug loading core of the micelles. Despite the virtual water-soluble feature of PVP-VA, the employment of PEO-VA-NVCL made the bi-block polymer a real amphipathic micelle material as predicted by its chemical structure, with PVP fragment working as hydrophilic part and VA fragment working as hydrophobic part. Then, a question occurred to us: why was PVP-VA able to entrap the compound and form the drug loading micelles instead of dissolving in water completely? Obviously, the answer to the question lies with the tri-block polymer PEOVA-NVCL with the interfacial layer formation ability. As was discussed earlier, the NVCL fragment located at the interface of the micelles, separating the PEO shell and VA core. Although the NVCL signals were too weak to be distinguished from that of PVP (Figure 4A upper), it was supposed that NVCL fragment constructed the interfacial layer of the drug loading micelles. Due to the excellent micelle formation ability of the tri-block polymer, PEO-VA-NVCL transformed into micelle structure as soon as the NM-SD started to dissolve, and at the same time, the interfacial layer of NVCL fragment not only encapsulated the compound together with the VA fragment of the tri-block polymer itself, but also the hydrophobic fragment of PVP-VA. As a result, with the help of the interfacial layer of NVCL fragment, PEO and 
PVP constructed the shell of the micelles and, VA fragment of both polymers together with the compound constructed the core of the micelles. In a word, the interfacial layer of the NVCL fragment successfully "prisoned" the compound as well as the VA fragment of PVP-VA which intended to "escape" into water (Figure 4B).

Actually, other amphipathic polymers with hydrophilic and hydrophobic fragments only failed in preventing the escape of the seemingly amphipathic polymer PVP-VA, such as PEG-PLA, PEG-PCL, and so on (data not shown). The results highlighted the significance of the interfacial layer of NVCL fragment in stabilizing micelles and indicated that the tri-block polymer PEO-VA-NVCL with interfacial layer formation ability seemed to be a potential micelle candidate with excellent performances.

\section{Conclusion and discussion}

In the present research, a unique tri-block polymer with NVCL fragment working as interfacial layer of the micelles and separating the outside hydrophilic PEO shell and the inner hydrophobic core was suggested. The polymer exhibited satisfactory micelle constructing capacity in the terms of organic solvent-free procedure and rapid micelle formation as soon as contacting water. When PEO-VA-NVCL was introduced as co-excipient in the formulation of NM-SD, the problem of drug recrystallization in the release media or body fluids which retarded the practical application of SD in enhancing oral bioavailability of hardly soluble drugs was completely solved. Drug loading micelles were found in the dissolution media, accounting for the maintenance of the supersaturated solution of the compound.

In the present study, nanomaterial was employed to solve the problem puzzling the application of SD. More importantly, a tri-block polymer exhibiting outstanding performances in constructing nanodrug delivery systems was proposed.

Nowadays, polymers with different water soluble and water insoluble fragments have been synthesized. These polymers are often used to entrap hardly soluble compounds, with hydrophobic fragments forming payload containing core and hydrophilic fragments constructing shell.

These polymers have one character in common, ${ }^{4-29}$ the fragments are either water soluble to form hydrophilic shell or water insoluble to shape hydrophobic core. In this paper, a tri-block polymer named PEO-VA-NVCL was proposed. Apart from the hydrophilic part of PEO and hydrophobic fraction of VA, the polymer has the third fragment of
NVCL with intermediate solubility to construct a special interfacial layer.

Unlike other reported polymers, PEO-VA-NVCL was able to form micelle solution simply by slowly stirring the polymer granules in water for several minutes. This organicsolvents free feature is likely to simplify the preparation of micelle formulations and make large-scale manufacture of nanodrug delivery systems a reality.

As is known to all, the stability of micelles in blood circulation remains a great concern. In detail, it is supposed that drugs might "escape" from hydrophobic core of normal micelles and, in some cases, the micelle particles are likely to totally disintegrate in vivo. It was found in the current research that the interfacial layer constructed by NVCL fragment succeeded in "prisoning" the VA fragment of PVP-VA which otherwise would dissolve in water. As a result, PVP-VA together with a small amount of PEOVA-NVCL constructed stable micelles which entrapped the compound safely. In other words, PEO-VA-NVCL at a low dose successfully stabilized the micelles comprising PVP-VA and the drug, which would otherwise disintegrate. This kind of disintegration in vitro is similar to the disintegration that may happen to the micelles in the blood circulation. The fact that the PEO-VA-NVCL rather than other amphipathic polymers stabilized the NM/PVP-VA micelles and prevented the disintegration implied the importance of interfacial layer in producing stable drug loading micelles. Based on this point, there is a possibility that micelles constructed by PEO-VA-NVCL are more likely to resist the destructions and keep its integrity in blood circulation than micelles constructed by normal amphipathic polymers. Actually, the hemolysis rate was $<3 \%$ even if the concentration of the triblock polymer in the blood sample was $1 \mathrm{mg} / \mathrm{ml}$ (Figure 5A and B), and the hemolysis rate was comparable to that of the negative control blood sample in PBS. Furthermore, Figure 5C shows that the particle size of the tri-block polymer micelles from the supernatants of the hemolysis test samples was similar to that of the original polymer solution without blood cells, both of them were about $62 \mathrm{~nm}$ (Figure 5C). The above results not only implied that the toxicity of the tri-block polymer was negligible, but also indicated that the micelle structure remained intact in the circulatory system. As the compatibility and the stability of the nanocarriers are of great importance to its use in the circulatory system, the above phenomena clued the possible widespread applications of the tri-block polymer in the future. 


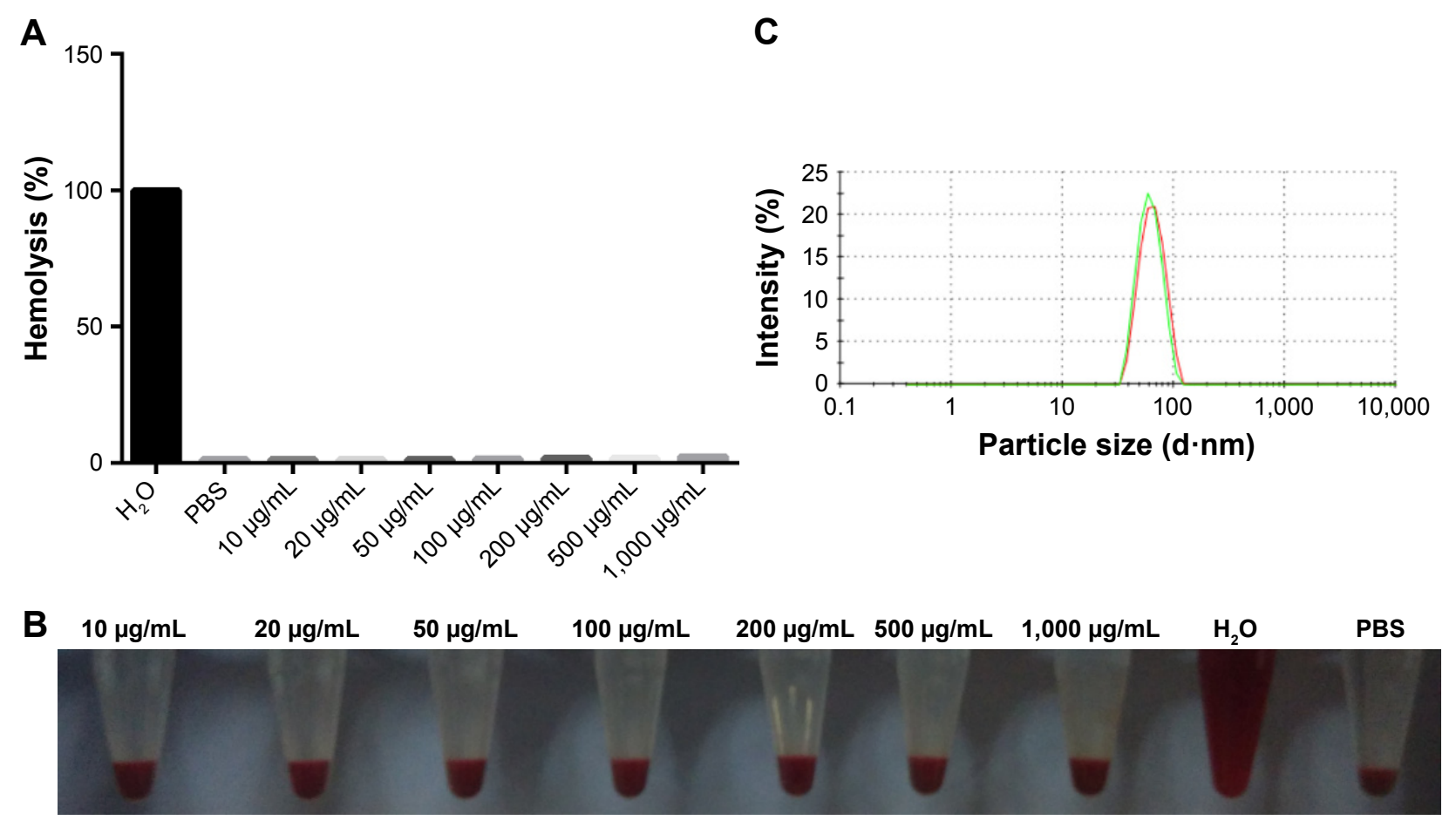

Figure $\mathbf{5}$ The interactions between the tri-block polymer micelles and the blood cells. (A) The hemolysis rate of the rat blood cells after incubating in the micelle solutions with different micelle concentrations for I day. (B) Images of the hemolysis test. (C) Particle size of the original tri-block polymer micelles (red) and that of the supernatant of the hemolysis sample (green).

Abbreviation: PBS, phosphate-buffered saline.

\section{Acknowledgments}

The authors would like to thank Professor Bo Wu, Dr LingRan Du, and Dr Yu-Gang Huang for their help in editing the paper. The research was supported by funding from the Scientific Research Foundation Project of Bureau of Education of Guangzhou (grant numbers 1201410511 and 1201610085), the National Natural Science Foundation (grant number 51403043), the Guangdong Natural Science Foundation Project (grant number 2015A030310037) the Natural Science Foundation Project of Guangzhou Medical University (grant number 2014C09), and the Guangdong Medical Science \& Technology Research Foundation Project (grant number A2017069).

\section{Disclosure}

The authors report no conflicts of interest in this work.

\section{References}

1. Jijun F, Lili Z, Tingting G, Xing T, Haibing H. Stable nimodipine tablets with high bioavailability containing NM-SD prepared by hot-melt extrusion. Powder Technol. 2010;204(2-3):214-221.

2. Jijun F, Lishuang $X$, Xiaoli W, et al. Nimodipine (NM) tablets with high dissolution containing NM solid dispersions prepared by hot-melt extrusion. Drug Dev Ind Pharm. 2011;37(8):934-944.

3. Jijun F, Lishuang X, Xiaoguang T, et al. The inhibition effect of high storage temperature on the recrystallization rate during dissolution of nimodipine-Kollidon VA64 solid dispersions (NM-SD) prepared by hot-melt extrusion. J Pharm Sci. 2011;100(5):1643-1647.
4. Li S, Li C, Jin S, et al. Overcoming resistance to cisplatin by inhibition of glutathione-S-transferases (GSTs) with ethacraplatin micelles in vitro and in vivo. Biomaterials. 2017;144:119-129.

5. Garg SM, Paiva IM, Vakili MR, et al. Traceable PEO-poly(ester) micelles for breast cancer targeting: the effect of core structure and targeting peptide on micellar tumor accumulation. Biomaterials. 2017; 144:17-29.

6. Wang L, Liu X, Zhou Q, et al. Terminating the criminal collaboration in pancreatic cancer: nanoparticle-based synergistic therapy for overcoming fibroblast-induced drug resistance. Biomaterials. 2017;144:105-118.

7. Guo D, Xu S, Wang N, et al. Prodrug-embedded angiogenic vesseltargeting nanoparticle: a positive feedback amplifier in hypoxia-induced chemo-photo therapy. Biomaterials. 2017;144:188-198.

8. Zhao N, Wu B, Hu X, Xing D. NIR-triggered high-efficient photodynamic and chemo-cascade therapy using caspase-3 responsive functionalized upconversion nanoparticles. Biomaterials. 2017;141:40-49.

9. Roy A, Zhao Y, Yang Y, Szeitz A, Klassen T, Li SD. Selective targeting and therapy of metastatic and multidrug resistant tumors using a long circulating podophyllotoxin nanoparticle. Biomaterials. 2017; 137:11-22.

10. Aji Alex MR, Nehate C, Veeranarayanan S, Kumar DS, Kulshreshtha R, Koul V. Self assembled dual responsive micelles stabilized with protein for co-delivery of drug and siRNA in cancer therapy. Biomaterials. 2017; 133:94-106.

11. Gao M, Fan F, Li D, et al. Tumor acidity-activatable TAT targeted nanomedicine for enlarged fluorescence/magnetic resonance imagingguided photodynamic therapy. Biomaterials. 2017;133:165-175.

12. Li F, Li T, Cao W, Wang L, Xu H. Near-infrared light stimuliresponsive synergistic therapy nanoplatforms based on the coordination of tellurium-containing block polymer and cisplatin for cancer treatment. Biomaterials. 2017;133:208-218.

13. Kim MJ, Lee Y, Jon S, Lee DY. PEGylated bilirubin nanoparticle as an anti-oxidative and anti-inflammatory demulcent in pancreatic islet xenotransplantation. Biomaterials. 2017;133:242-252. 
14. Tang L, Zhang F, Yu F, et al. Croconaine nanoparticles with enhanced tumor accumulation for multimodality cancer theranostics. Biomaterials. 2017;129:28-36.

15. Feliciano CP, Tsuboi K, Suzuki K, Kimura H, Nagasaki Y. Long-term bioavailability of redox nanoparticles effectively reduces organ dysfunctions and death in whole-body irradiated mice. Biomaterials. 2017; 129:68-82.

16. Jin W, Wang Q, Wu M, et al. Lanthanide-integrated supramolecular polymeric nanoassembly with multiple regulation characteristics for multidrug-resistant cancer therapy. Biomaterials. 2017;129:83-97.

17. Logie J, Ganesh AN, Aman AM, Al-Awar RS, Shoichet MS. Preclinical evaluation of taxane-binding peptide-modified polymeric micelles loaded with docetaxel in an orthotopic breast cancer mouse model. Biomaterials. 2017;123:39-47.

18. Wang Q, Jiang H, Li Y, et al. Targeting NF-kB signaling with polymeric hybrid micelles that co-deliver siRNA and dexamethasone for arthritis therapy. Biomaterials. 2017;122:10-22.

19. Zeng Q, Li H, Jiang H, et al. Tailoring polymeric hybrid micelles with lymph node targeting ability to improve the potency of cancer vaccines. Biomaterials. 2017;122:105-113.

20. Tian X, Yang F, Yang C, et al. Toxicity evaluation of Gd2O3@SiO2 nanoparticles prepared by laser ablation in liquid as MRI contrast agents in vivo. Int J Nanomedicine. 2014;9:4043-4053.

21. Luo CF, Hou N, Tian J, et al. Metabolic profile of puerarin in rats after intragastric administration of puerarin solid lipid nanoparticles. Int $J$ Nanomedicine. 2013;8:933-940.
22. Luo CF, Yuan M, Chen MS, et al. Pharmacokinetics, tissue distribution and relative bioavailability of puerarin solid lipid nanoparticles following oral administration. Int J Pharm. 2011;410(1-2): $138-144$.

23. Xia Y, Xu T, Wang C, et al. Novel functionalized nanoparticles for tumor-targeting co-delivery of doxorubicin and siRNA to enhance cancer therapy. Int J Nanomedicine. 2018;13:143-159.

24. Li Y, Lin Z, Guo M, et al. Inhibitory activity of selenium nanoparticles functionalized with oseltamivir on HINI influenza virus. Int J Nanomedicine. 2017;12:5733-5743.

25. Li Y, Guo M, Lin Z, et al. Polyethylenimine-functionalized silver nanoparticle-based co-delivery of paclitaxel to induce HepG2 cell apoptosis. Int J Nanomedicine. 2016;11:6693-6702. eCollection 2016.

26. Wang H, Yin H, Yan F, et al. Folate-mediated mitochondrial targeting with doxorubicin-polyrotaxane nanoparticles overcomes multidrug resistance. Oncotarget. 2015;6(5):2827-2842.

27. Yang RM, Fu CP, Fang JZ, et al. Hyaluronan-modified superparamagnetic iron oxide nanoparticles for bimodal breast cancer imaging and photothermal therapy. Int J Nanomedicine. 2017;12:197-206.

28. Zhang R, Lin Z, Lui VCH, et al. Silver nanoparticle treatment ameliorates biliary atresia syndrome in rhesus rotavirus inoculated mice. Nanomedicine. 2017;13(3):1041-1050.

29. Lin L, Cai M, Deng S, et al. Amelioration of cirrhotic portal hypertension by targeted cyclooxygenase-1 siRNA delivery to liver sinusoidal endothelium with polyethylenimine grafted hyaluronic acid Nanomedicine. 2017;13(7):2329-2339.
International Journal of Nanomedicine

\section{Publish your work in this journal}

The International Journal of Nanomedicine is an international, peerreviewed journal focusing on the application of nanotechnology in diagnostics, therapeutics, and drug delivery systems throughout the biomedical field. This journal is indexed on PubMed Central, MedLine, CAS, SciSearch $\AA$, Current Contents $₫ /$ Clinical Medicine,

\section{Dovepress}

Journal Citation Reports/Science Edition, EMBase, Scopus and the Elsevier Bibliographic databases. The manuscript management system is completely online and includes a very quick and fair peer-review system, which is all easy to use. Visit http://www.dovepress.com/ testimonials.php to read real quotes from published authors. 